\title{
Thermodynamically stable noncomposite vortices in mesoscopic two-gap superconductors
}

\author{
L. F. Chibotaru, V. H. Dao and A. Cenlemans
}

Division of Quantum and Physical Chemistry and INPAC, Institute for Nanoscale Physics and Chemistry, University of Leuven - Celestijnenlaan, 200F, B-3001 Leuven, Belgium

received 6 March 2007; accepted in final form 5 April 2007

published online 3 May 2007

PACS 74.78.Na - Mesoscopic and nanoscale systems

PACS 74.20.De-Phenomenological theories (two-fluid, Ginzburg-Landau, etc.)

PACS 74.25. Ha - Magnetic properties

\begin{abstract}
In mesoscopic two-gap superconductors with sizes of the order of the coherence length noncomposite vortices are found to be thermodynamically stable in a large domain of the $T-H$ phase diagram. In these phases the vortex cores of one condensate are spatially separated from the other condensate ones, and their respective distributions can adopt distinct symmetries. The appearance of these vortex phases is caused by a non-negligible effect of the boundary of the sample on the superconducting order parameter and represents therefore a genuine mesoscopic effect. For low values of interband Josephson coupling vortex patterns with $L_{1} \neq L_{2}$ can arise in addition to the phases with $L_{1}=L_{2}$, where $L_{1}$ and $L_{2}$ are total vorticities in the two condensates. The calculations show that noncomposite vortices could be observed in thin mesoscopic samples of $\mathrm{MgB}_{2}$.
\end{abstract}

Copyright (C) EPLA, 2007

Two-gap superconductivity started to attract much attention in connection with the discovery of the $\mathrm{MgB}_{2}$ superconductor, for which a clear-cut evidence for the existence of two gaps was obtained [1]. Superconductors of this kind show new qualitative effects with respect to conventional ones. First, the presence of two or more electronic bands at the Fermi level always enhances the superconductivity as compared to the effect from any individual band. Second, a new phenomenon, the fractionalization of the magnetic flux associated to individual vortices in massive two-gap superconductors is predicted $[2,3]$. The condition for this fractionalization is the inequality of the winding numbers of the vortices in the two condensates $\left(L_{1} \neq L_{2}\right)$ having a common vortex core (composite vortices). Although these vortex phases have finite energies per unit length they never correspond to the ground state, i.e. are thermodynamically unstable $[2,3]$. On the other hand, the energy per unit length of a vortex configuration where the vortices in each of the two bands are spatially separated (noncomposite or deconfined vortices) was found to be divergent [2]. These results are in line with the fact that only composite vortices with $L_{1}=L_{2}=1$, i.e. usual Abrikosov vortices, have been observed in massive two-gap superconductors to date.

Fractionalization of the vortex magnetic flux can also be observed in layered superconductors through thermal fluctuations [4]. By the action of the interlayer Josephson coupling and the magnetic field, pancake vortices (which individually live inside one layer) align themselves into vortex stacks which thread across the layers. Since each pancake vortex carries only $\approx \Phi_{0} / N_{l}$ (where $N_{l}$ is the number of layers) the dissociation of a vortex stack results in a net fraction of the flux quantum. This mechanism is however different from the one in a multigap superconductor where a fractional flux vortex lives within one condensate but threads through the entire sample thickness.

In mesoscopic superconductors, the geometry of the confinement of the superconducting condensate influences essentially their properties because the coherence length, $\xi(T)$, and the penetration length, $\lambda(T)$, become of the order of the samples size (for a review, see, e.g., $[5,6]$ ). In particular, new vortex patterns arise as a function of boundary geometry leading to cusp-like normalsuperconducting phase boundary, $T_{c}(H)[6,7]$, and to the nucleation of giant vortices in disks $[8,9]$ and of antivortices in regular polygons $[6,10]$. Another important mesoscopic effect is that the vortices nucleate in patterns which are quite different from an Abrikosov lattice and approach the latter by a series of phase transitions when temperature is lowered from $T_{c}(H)[9,11]$. The symmetry of nucleated vortex patterns is always consistent with the geometry of the boundary of the sample [6], while the region of their 
stability against Abrikosov-type vortices increases with diminishing size of the sample, persisting down to $T=0$ when the samples reach some critical size [11].

In this letter we investigate the vortex patterns in thin mesoscopic two-gap superconductors. Contrary to what was found in the case of massive superconductors, we show that noncomposite vortices can arise as thermodynamically stable phases in mesoscopic samples of small enough size. We find the reasons for their stabilization and discuss the possibility of their experimental observation.

Consider a superconducting sample of size $R$ and thickness $d$ in a perpendicular uniform magnetic field $H$. For small $(R \sim \xi)$ and thin $(d \ll \xi<\lambda)$ samples one can neglect the variation of the order parameter across thickness and the distortion of the magnetic field induced by screening and vortex currents ${ }^{1}[6,7,9]$. The two components of the order parameter $\Psi_{1,2}[12]$ are found from the minimization of the following 2D Ginzburg-Landau functional [13]:

$$
\begin{aligned}
\Delta F= & \int\left[\sum_{n=1}^{2}\left(\frac{1}{2 m_{n}}\left|\left(-i \hbar \nabla-\frac{2 e}{c} \mathbf{A}\right) \Psi_{n}\right|^{2}\right)+\alpha_{n}\left|\Psi_{n}\right|^{2}\right. \\
& \left.\left.+\frac{1}{2} \beta_{n}\left|\Psi_{n}\right|^{4}\right)-\gamma\left(\Psi_{1}^{*} \Psi_{2}+\Psi_{2}^{*} \Psi_{1}\right)\right] \mathrm{d} S
\end{aligned}
$$

where $\mathbf{A}$ is the vector potential of the applied field, $\alpha_{1}=-a_{1} t$ and $\alpha_{2}=\alpha_{20}-a_{2} t$ are the condensation energy parameters for the active and the passive band, respectively, $t \approx 1-T / T_{1}$, and $T_{1}$ is the critical temperature corresponding to the active band [13]. At the normal/superconducting phase transition, minimizing (1) (without the terms $\sim\left|\Psi_{n}\right|^{4}$ ) results in two linear equations describing the nucleation of superconductivity and two boundary conditions:

$$
\begin{aligned}
& {\left[\alpha_{n}+\frac{1}{2 m_{n}}\left(-i \hbar \nabla-\frac{2 e}{c} \mathbf{A}\right)^{2}\right] \Psi_{n}-\gamma \Psi_{n^{\prime}}=0,} \\
& \left(-i \hbar \nabla-\frac{2 e}{c} \mathbf{A}\right) \Psi_{\left.n\right|_{\text {n. b. }}}=0,
\end{aligned}
$$

where $n, n^{\prime}=1,2$ and 2,1 . The notation $\left.\right|_{\text {n.b. means the }}$ expression is projected on the unit vector normal to the boundary. It is straightforward to show that the nucleation solution of these equations has the form $\Psi_{1} \sim \Psi_{2} \sim \phi_{N}$, where $\phi_{N}$ is the solution of the eigenvalue equation:

$$
\begin{aligned}
& \left(-i \hbar \nabla-\frac{2 e}{c} \mathbf{A}\right)^{2} \phi_{i}=\lambda_{i} \phi_{i} \\
& \left(-i \hbar \nabla-\frac{2 e}{c} \mathbf{A}\right) \phi_{\left.i\right|_{\text {n. b. }}}=0
\end{aligned}
$$

corresponding to $\lambda_{N}(H)$ which is the lowest of the eigenvalues $\lambda_{i}(H)$. This eigenvalue determines the nucleation

\footnotetext{
${ }^{1}$ For a thin superconductor with $\Psi(\mathbf{r})=\sqrt{\frac{|\alpha|}{\beta}} f(\mathbf{r}) e^{i \chi(\mathbf{r})}$, the variation of the vector potential due to the supercurrent $\delta \mathbf{A}\left(\mathbf{r}_{0}\right) \approx$ $-\frac{d}{4 \pi \lambda^{2}} \int\left(\frac{\Phi_{0}}{2 \pi} \nabla \chi+\mathbf{A}\right) f^{2}(\mathbf{r}) /\left|\mathbf{r}_{0}-\mathbf{r}\right| \mathrm{d} S \sim-\frac{d R}{2 \lambda^{2}}\left(\frac{\Phi_{0}}{2 \pi} \nabla \chi+\mathbf{A}\right) f^{2}\left(\mathbf{r}_{0}\right)$ can be neglected if the thickness $d$ is small enough. The same argument can be generalized to a two-gap superconductor.
}

phase boundary $T_{c}(H)$ [13] and the ratio of the amplitudes of the two components of the order parameter, $\Psi_{2} / \Psi_{1}=\gamma /\left(\alpha_{2}+\lambda_{N} / 2 m_{2}\right)$. Thus the distribution of the nucleated order parameter in the two bands of a two-gap superconductor is described by the same function.

The eigenstates of (3) are then used as the basis set for the order parameter of the complete functional (1),

$$
\Psi_{1}=\sum_{i} u_{i} \phi_{i}, \quad \Psi_{2}=\sum_{i} v_{i} \phi_{i},
$$

which yields $\Delta F$ as a function of the expansion coefficients $\left\{u_{i}, v_{i}\right\}$.

We will consider further a disk of radius $R$. It is then convenient to introduce new "lengths" defined by the relations: $-\alpha_{1}=\hbar^{2} / 2 m_{1} \xi_{1}(T)^{2}, \alpha_{20}=\hbar^{2} / 2 m_{1} \xi_{20}^{2},-\alpha_{2} t=$ $\hbar^{2} / 2 m_{1} \xi_{2}(T)^{2}$, and $\gamma=\hbar^{2} / 2 m_{1} \xi_{\gamma}^{2}$. When $\gamma=0$ and $\alpha_{2}>0$ the superconductivity nucleates in the active band, for which the coherence length is $\xi_{1}$. The order parameter corresponds to one single giant vortex in the center of the disk [8] whose winding number increases with the applied field following the cusps on the nucleation phase boundary line (fig. 1a). At lower temperatures it undergoes a broken-symmetry phase transition to a multivortex state corresponding to Abrikosov $(L=1)$ vortices forming a regular polygon [9]. Since $1 / \xi_{1}^{2} \sim T_{c}-T$, it follows that the temperature region where the nucleated (giant vortex) phase is thermodynamically stable (dark regions in fig. 1a) scales with the size of the disk as $\sim R^{-2}$. Note that for $R \geqslant 6 \xi_{1}$ new broken-symmetry phases arise, which correspond to off-center displacements of the central vortex in the case $L=1$ and of the maximum of $|\Psi|^{2}$ distribution in the case $L=0$ [14].

In the case of nonzero interband Josephson coupling similar phase diagrams emerge (fig. 1b). We see again regions corresponding to giant vortex patterns (shown in blue in fig. 1b), with the same vortex numbers in both condensates, and regions with broken-symmetry vortex patterns (yellow and pink regions in fig. 1b) consisting of Abrikosov vortices. However, a qualitatively new feature arises in the broken-symmetry phases, with two vortex patterns, corresponding to $\Psi_{1}$ and $\Psi_{2}$, being spatially distinct for any parameters of (1). Therefore each vortex in these phases is a noncomposite vortex. Moreover, in contrast to single band superconductors (fig. 1a), we have now two types of multivortex phases, corresponding to $L_{1}=L_{2}$ (yellow regions in fig. 1 b) and $L_{1} \neq L_{2}$ (pink regions in fig. 1b). The corresponding order parameter's density plots are shown in fig. 2 .

The temperature evolution of the vortex patterns in two condensates is strongly dependent on the parameter $\alpha_{2}$, while the difference in their shape increases with diminishing $\gamma$. Indeed, as fig. 2 shows with $R / \xi_{\gamma}(0)=0.1$, for small values of $\gamma$ we encounter three qualitatively different situations. In the first case (a) the transition to the broken-symmetry phase leads to a strong separation of the multivortex patterns, corresponding to the active (green) and the passive (red) bands, the latter approaching 


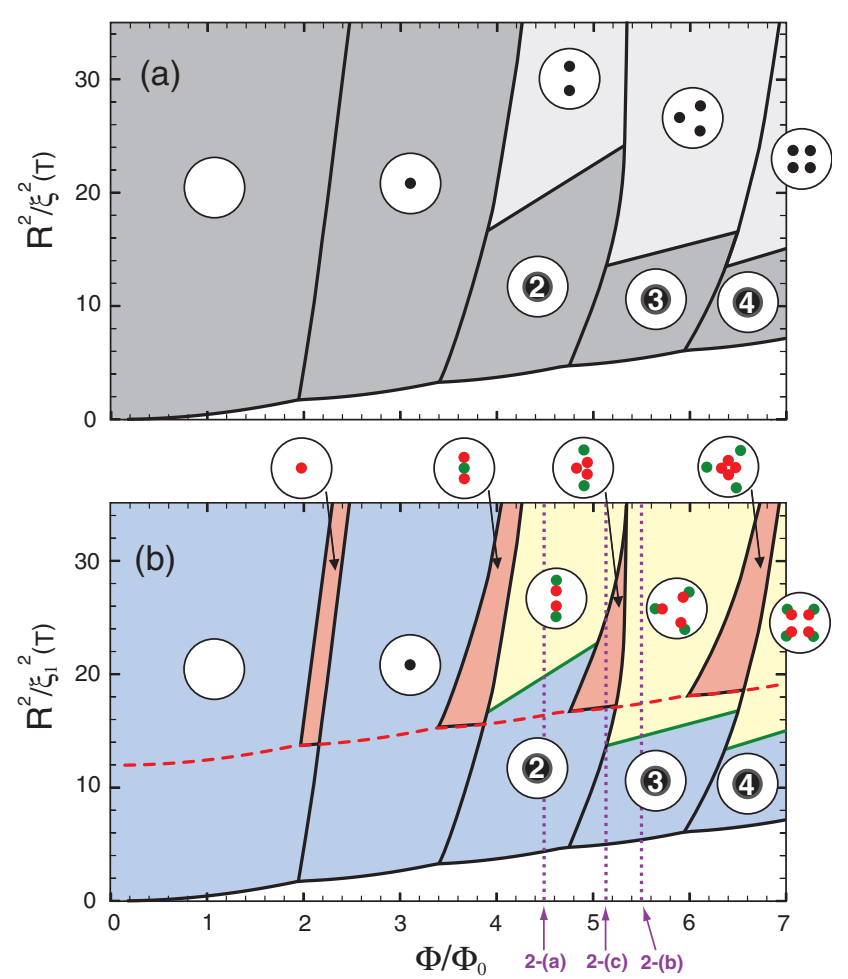

Fig. 1: Phase diagram for a thin disk of single-gap (a) and twogap (b) superconductors. The phase diagram (a) is parameterfree [11], while the diagram (b) is for $a_{1} / a_{2}=\beta_{1} / \beta_{2}=$ $m_{1} / m_{2}=1, R / \xi_{2}(0)=2 \sqrt{3}$ and $R / \xi_{\gamma}(0)=0.1$. For each phase, the vortex structure is shown schematically in black for composite vortices and in green (active band) and red (passive band) for noncomposite ones. The numbers inside the circles indicate the vorticities of the giant vortex phases. Vertical lines separate phases with different total vorticity $L$. Continuous horizontal lines correspond to broken-symmetry phase transitions, which are of giant vortex - multivortex type for $L>1$. The dashed line is the nucleation phase boundary for the passive band for $\gamma=0$ (see the text). The vertical dotted lines mark the positions of the three graphics in fig. 2 .

the first one with further lowering of temperature. In the second case (b) the vortex pattern in the passive band first shrinks, resulting in its strong separation from the vortex pattern in the active band, and then approaches it again when the temperature is lowered. Finally, the case (c) corresponds to the transition to the brokensymmetry phase phase with $L_{1} \neq L_{2}$, followed by the transition to a broken-symmetry phase with $L_{1}=L_{2}$ when the temperature is lowered further.

The reason for the above behaviour of the order parameter can be elucidated by the following simple consideration [11]. Let us suppose for simplicity that only one eigenstate of $(3)\left(\sim \phi_{A}\right)$ admixes to the nucleated order parameter $\left(\sim \phi_{N}\right)$ in the point of broken-symmetry transition, which is actually a reasonable approximation for disks [9] and regular polygons [11]. When $\gamma$ is small the nucleation of superconductivity takes place at $-\alpha_{1} \approx \lambda_{N} / 2 m_{1}$. At the onset of the transition to the

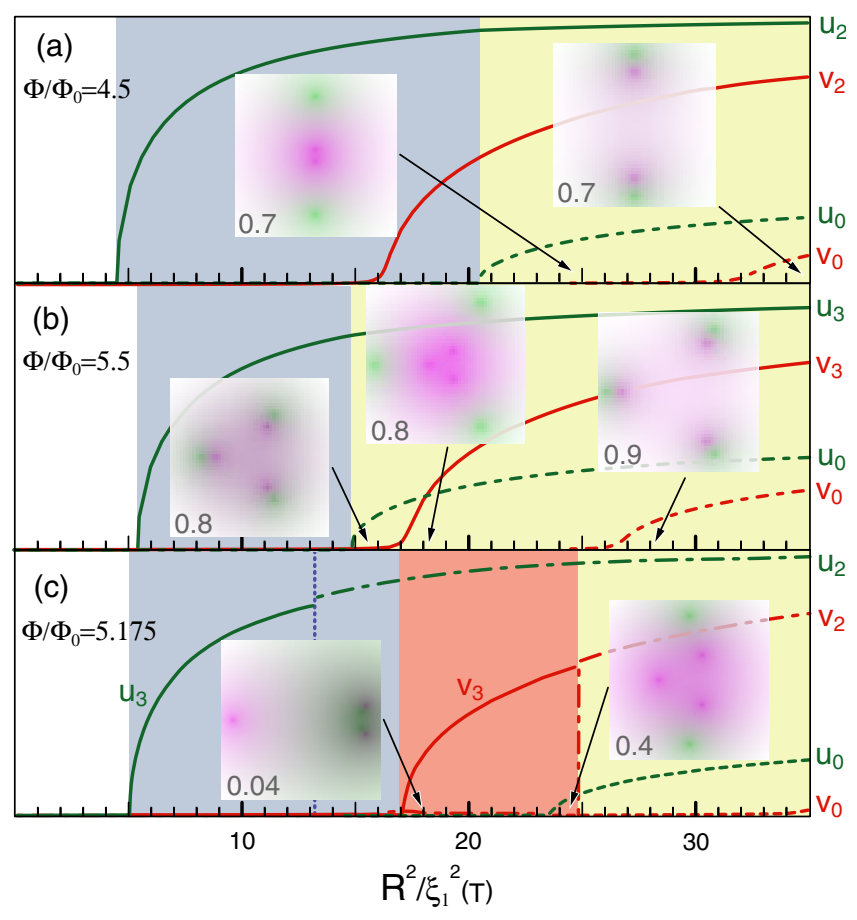

Fig. 2: (a) Evolution of the order parameter for three values of the applied magnetic flux in the phase diagram of fig. $1 \mathrm{~b}$. The background colours correspond to the giant vortex phases (blue) and the multivortex phases with $L_{1}=L_{2}$ (yellow) and $L_{1} \neq L_{2}$ (pink). The expansion coefficients $u_{L}$ and $v_{L}$ correspond to the ground state solutions of (3) for each $L$ that contribute substantially to the expansion. The numbers in the corner of inserted plots gives the side of the zoom on the disk in units of $R$. The increase of the intensity of colors corresponds to the decrease of the density of the order parameters, so that the darkest regions denote the position of the vortex cores.

broken-symmetry phase we have $\Psi_{1}=u_{N} \phi_{N}+u_{A} \phi_{A}\left(\phi_{A}\right.$ is supposed to be of different symmetry compared to $\phi_{N}$, which in the case of disk implies different rotational quantum numbers). If $\alpha_{2}+\lambda_{N} / 2 m_{2}>0$ (the sufficient condition for this is $\left.\xi_{2}(0)>\xi_{20}\right)$, the minimization of (1) gives for the expansion coefficients of $\Psi_{2}$ :

$$
v_{i} \approx \frac{\beta_{1}}{\beta_{2}} \frac{\xi_{1}^{2}}{\xi_{\gamma}^{2}} \frac{1}{\frac{\xi_{1}^{2}}{\xi_{20}^{2}}-\frac{\xi_{1}(0)^{2}}{\xi_{2}(0)^{2}}+\frac{m_{1}}{m_{2}} \frac{\xi_{1}^{2}}{R^{2}} \epsilon_{i}} u_{i},
$$

where $\epsilon_{i}=\lambda_{i} R^{2} / \hbar^{2}$ are dimensionless eigenvalues of (3), only $\xi_{1}$ is temperature dependent, and the dependence on $R$ is explicit. If $v_{i}$ and $u_{i}$ were related by the same proportionality coefficient, the order parameters $\Psi_{1}$ and $\Psi_{2}$ would have been described by essentially the same function, in particular, all vortices arising in such a phase would correspond to composite ones. Such a situation obviously occurs in the giant vortex phase. However, as eq. (5) shows, the transition to a broken-symmetry phase makes the coefficients of proportionality different for the admixed $(i=A)$ and the nucleated $(i=N)$ components of the order parameter due to the term $\sim \epsilon_{i}$ in the denominator of (5). This term becomes important for small values 
of $R$, i.e. in the mesoscopic regime, and it disappears in the macroscopic regime $R \rightarrow \infty$. This is in line with the conclusion mentioned in the introduction, that only composite vortices can arise in thermodynamically stable phases of massive two-gap superconductors.

The above analysis allows to explain the phase diagram in fig. 1b. For large positive $\alpha_{2}+\lambda_{N} / 2 m_{2}$ the superconductivity in the passive band is induced by the Josephson coupling to the active band. Then the order parameter and the resulting vortex pattern in the passive band follow closely that of the active band, according to eq. (5). However, when this quantity is small and can turn to negative at some temperature $T^{*}$ (shown by dashed line in fig. 1b) then for $T<T^{*}$ the nucleated component $\phi_{N}$ in the passive band begins to grow much faster than in the previous case due to the intrinsic superconductivity which now exists in the passive band. Then two qualitative situations can occur. If at $T=T^{*}$ the superconductor is still in the giant vortex phase, the subsequent transition to the multivortex phase in the active band will not induce a similar order parameter in the passive band. The latter will remain almost $\sim \phi_{N}$ and, consequently, a much smaller amplitude of the splitting of the giant vortex into Abrikosov vortices is expected (fig. 2a). If at $T=T^{*}$ the system is already in the multivortex state, then the subsequent lowering of temperature will induce the shrinkage of the vortex pattern in the passive band due to a faster stabilization of the nucleated component in $\Psi_{2}$. This is exactly what is seen in fig. 2b. In both these situations a strong spatial separation of the vortex patterns is achieved. The existence of the vortex patterns with $L_{1} \neq L_{2}$ is merely due to the fact that for $\gamma=0$ the phase diagram for the passive band is shifted upwards relative to the active one (the nucleation of superconductivity takes place at lower temperatures), which creates overlap regions where the two condensates have different vorticities. If $\gamma$ is smaller than the difference of free energies corresponding to $L_{1}$ and $L_{2}$ in the passive band, then vortex patterns with different vorticities in the condensates will be stabilized also for $\gamma \neq 0$, but will disappear from the phase diagram when $R / \xi_{\gamma}$ exceeds some critical value. The existence of the overlap regions with $L_{1} \neq L_{2}$ is possible due to the fact that the lines separating domains of different vorticity on the diagram in fig. 1 are not vertical. As a consequence, lowering the temperature at fixed applied flux can result in several phase transitions with change of vorticity in the condensates, accompanied by jumps of the order parameter, i.e. of the coefficients $u_{i}$ and $v_{i}$ in eq. (4) (fig. 2c).

Finally, we calculate the $T-H$ phase diagram for a thin disk of $\mathrm{MgB}_{2}$ with the anisotropy axis $c$ perpendicular to the plane of the sample ${ }^{2}$ (fig. 3). Formation of noncomposite vortices is favored by a weak interband coupling $\gamma$ and a small mass ratio $m_{2} / m_{1}$. From current available data, the smallest $\gamma$ is obtained for a sample

${ }^{2}$ This is the way in which thin $\mathrm{MgB}_{2}$ films actually grow on the substrate.

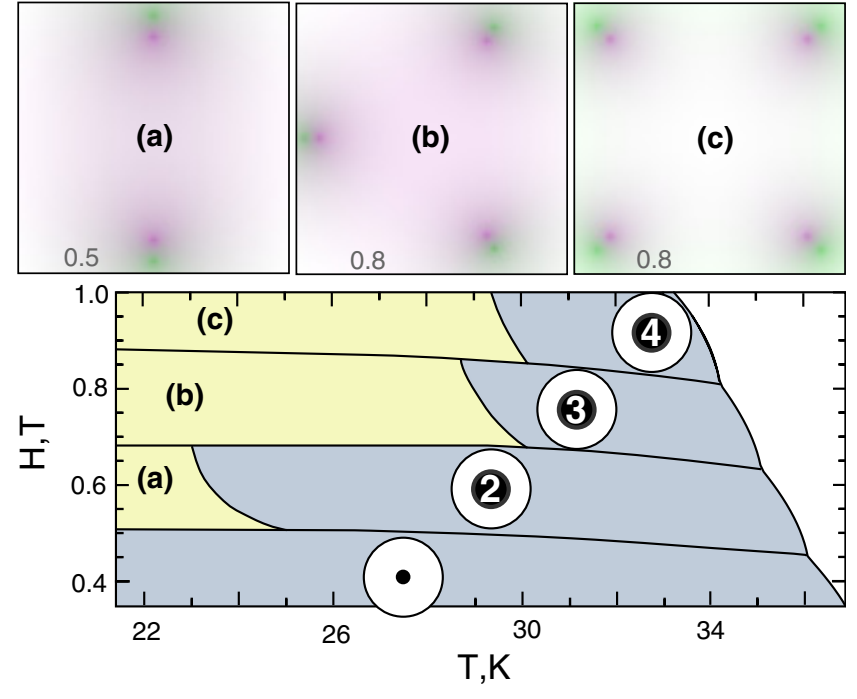

Fig. 3: The phase diagram for a thin disk of $\mathrm{MgB}_{2}$ with the radius $R=70 \mathrm{~nm}$ and the parameters taken from ref. $[13,16]$. The upper panels are zooms on the distribution of the order parameter in broken-symmetry (multivortex) phases. The conventions follow figs. 1 and 2 .

of boron isotope $\mathrm{Mg}^{10} \mathrm{~B}_{2}$ with a value reduced by $30 \%$ from usual $\mathrm{Mg}^{11} \mathrm{~B}_{2}$ [15], while $m_{\pi} / m_{\sigma}<0.1$ has been observed in irradiated samples [16] (where the introduced disorder should change effective masses but not the coupling constants). Modifying consequently the mass ratio in the Ginzburg-Landau parameters estimated previously for pristine $\mathrm{MgB}_{2}$ [13], we use $a_{2} / a_{1}=\beta_{2} / \beta_{1}=1.5$, $m_{2} / m_{1}=0.07, R / \xi_{1}(0)=10, R / \xi_{20}=8.1, R / \xi_{\gamma}=6.3$ and $T_{c}=39 \mathrm{~K}$ for fig. $3[17]$. We can see several regions on the phase diagram where the two vortex patterns are well separated in space, some of them being shown in figs. 3a-c. The radius of the disk was taken $R=70 \mathrm{~nm}$ but similar separations of the vortex patterns $(3-5 \mathrm{~nm})$ were found for a wide range of radii: $R=30-120 \mathrm{~nm}$. We have observed that other sets of parameters are not critical for the separation of the two vortex patterns except for the ratio $m_{2} / m_{1}$ : a smaller ratio leads to a higher separation.

The existence of noncomposite vortex patterns in twogap superconductors can be experimentally verified by combining different local probe techniques $[18,19]$. The discussed effects are not related to the symmetry of the samples but arise due to the non-negligible influence of the boundary of the sample on the two superconducting condensates. In this sense the emergence of noncomposite vortices found in the present study represents a true mesoscopic effect, not observable in infinite superconductors. We note in this connection that noncomposite vortices could also be stabilized in ultracold atomic Fermi gases in optical lattices or in single traps where the BCS to Bose-Einstein condensation transition takes place. These finite-size systems possess tunable interaction parameters which can give rise to multiband superconductivity [20] and are thus the subject of new promising experiments. 


\section{$* * *$}

We would like to thank V. V. MOSHCHALKOV for useful discussions. VHD acknowledges the financial support by the grant EF/05/005 (INPAC) from the University of Leuven.

\section{REFERENCES}

[1] Nagamatsu J. et al., Nature (London), 410 (2001) 63; Canfield P. C. and Crabtree G. W., Phys. Today, 56 (2003) (3) 34.

[2] Babaev E., Phys. Rev. Lett., 89 (2002) 067001; Nucl. Phys. B, 686 (2004) 397.

[3] Tanaka Y., Phys. Rev. Lett., 88 (2002) 017002; J. Phys. Soc. Jpn., 70 (2001) 2844.

[4] De Col A., Geshrenbein V. B. and Blatter G., Phys. Rev. Lett., 94 (2005) 097001.

[5] Moshchalkov V. V. et al., Handbook of Nanostructured Materials and Nanotechnology, edited by NALwA H. S., Vol. 3 (Academic, San Diego) 1999, p. 9.

[6] Chibotaru L. F. et al., J. Math. Phys., 46 (2005) 095108.

[7] Moshchalkov V. V. et al., Nature (London), 373 (1995) 319.

[8] Saint-James D., Phys. Lett. A, 15 (1965) 13; Fink H. J. and Presson A. G., Phys. Rev., 151 (1966) 219; Moshchalkov V. V., Qiu X. G. and Bruyndoncx V., Phys. Rev. B, 17 (1997) 11793.

[9] Schweigert V. A., Peeters F. M. and Singha Deo P., Phys. Rev. Lett., 81 (1998) 2783; Schweigert V. A. and Peeters F. M., Phys. Rev. B, 57 (1998) 13817.
[10] Chibotaru L. F. et al., Nature (London), 408 (2000) 833; Phys. Rev. Lett., 86 (2001) 1323; BonCA J. and Kabanov V. V., Phys. Rev. B, 65 (2002) 012509; Misko V. R. et al., Phys. Rev. Lett., 90 (2003) 147003.

[11] Chibotaru L. F., Teniers G., Ceulemans A. and Moshchalkov V. V., Phys. Rev. B, 70 (2004) 094505.

[12] These are actually the gap functions $\Delta_{1,2}(\mathbf{r})$ in ref. [13], respectively.

[13] Zhitomirsky M. E. and Dao V. H., Phys. Rev. B, 69 (2004) 054508; Dao V. H. and Zhitomirsky M. E., Eur. Phys. J. B, 44 (2005) 183.

[14] These phases have not been found in previous studies [9] because disks of smaller radii were considered.

[15] Walte A. et al., Phys. Rev. B, 73 (2006) 064501. The change in the boron mass is expected to modify the electron-phonon interaction. Yet the interband Josephson coupling has been found to be sample dependent for $\mathrm{Mg}^{10} \mathrm{~B}_{2}$. Although a different level of impurity-induced scattering is argued to explain the discrepancy, the origin of the interband-coupling variation remains an open question.

[16] Tarantini C. et al., Phys. Rev. B, 73 (2006) 134518.

[17] In $\mathrm{MgB}_{2}$, the London penetration depth at $0 \mathrm{~K}$ ranges from $40 \mathrm{~nm}$ to more than $300 \mathrm{~nm}$ (see Golubov A. A. et al., Phys. Rev. B, 66 (2002) 054524). With diffusivities $D_{\sigma}=6.2$ and $D_{\pi}=62\left(\mathrm{~cm}^{2} \mathrm{~s}^{-1}\right)$ used in the present letter, the GL penetration depth is $\lambda(26 \mathrm{~K})=54 \mathrm{~nm}$.

[18] Kanda A. et al., Phys. Rev. Lett., 93 (2004) 257002.

[19] Eskildsen M. R. et al., Phys. Rev. Lett., 89 (2002) 187003.

[20] Iskin M. and SÁ de Melo C. A. R., cond-mat/0603601 (2006). 